\title{
Behavioral Sleep Intervention for Adolescents with Autism Spectrum Disorder: a Pilot Study
}

Jenna R. van Deurs, Laurie K. McLay, Karyn G. France and Neville M. Blampied

University of Canterbury

Russell B. Lang

Texas State University

Jolene E. Hunter

University of Canterbury

Author Note.

Jenna R van Deurs, School of Health Sciences, University of Canterbury; Associate Professor Laurie McLay, School of Health Sciences, University of Canterbury; Associate Professor Karyn France, School of Health Sciences, University of Canterbury; Professor Neville Blampied, Psychology Department, University of Canterbury; Associate Professor Russell Lang, Clinic for Autism Research Evaluation and Support, Texas State University; Jolene Hunter, School of Health Sciences, University of Canterbury

This research was supported by funding from the Health Research Council, New Zealand.

Conflict of interest: Ms van Deurs, Associate Professor McLay, Associate Professor France, Professor Blampied, Associate Professor Lang and Ms Hunter declare no conflict of interest.

Correspondence concerning this article should be addressed to: Jenna van Deurs, School of Health Sciences, University of Canterbury, Private Bag 4800, Christchurch, 8140, New Zealand. Email: jenna.vandeurs@pg.canterbury.ac.nz.Phone: (+64) 277418251 


\begin{abstract}
Objectives: Sleep disturbances are a significant problem for individuals with autism spectrum disorder (ASD) across the lifespan; however; there is a paucity of research examining effective sleep interventions for adolescents with ASD. Although research has demonstrated individuals with ASD can be meaningfully engaged in their own intervention process, such engagement has not appeared in previous studies targeting sleep in adolescents with ASD. Methods: This study investigated the feasibility of including pre-adolescents and adolescents (ages 9 to 14 years) with ASD as active intervention agents within comprehensive, individualized treatments for sleep problems. Participants had a range of intellectual functioning but all produced spoken language. Outcomes were evaluated using single-case designs. Results: Data suggest intervention was effective in eliminating sleep disturbance for all participants. Improvements were maintained during 18-to-24-month follow-up. All three participants and their parents indicated a high degree of treatment satisfaction.
\end{abstract}

Conclusions: Findings illustrate the feasibility and potential benefit of including adolescents with ASD in the process of developing and implementing individualized behavioral interventions for sleep problems.

Keywords: autism spectrum disorder, sleep assessment, sleep treatment, adolescent, functional behavior assessment 


\section{Behavioral sleep intervention for adolescents with autism spectrum disorder: a Pilot study}

Difficulties initiating and maintaining sleep as well as other topographies of sleep disturbance are a common clinical problem for individuals with ASD and their families. In a study of 1518 children with autism spectrum disorder (ASD), Malow et al. (2016) found that 71\% had clinically significant sleep problems, a much higher rate than in samples of typically developing individuals (e.g., Elrod et al., 2016). Although the exact cause of sleep problems in people with ASD likely varies across individuals, previous research suggests their sleep is impacted by a complex interaction between physiological (e.g., dysregulated melatonin), environmental, and behavioral (e.g., inadvertent reinforcement of sleep-interfering behavior) variables (Richdale \& Schreck, 2009). A range of sleep problems have been identified in people with ASD including sleep onset delay, reduced total sleep time, reduced sleep efficiency, and daytime fatigue (Baker, Richdale, Short, \& Gradisar, 2013). These issues appear to be particularly problematic during adolescent years (Baker et al., 2013; Goldman, Richdale, Clemons, \& Malow, 2012). This is consistent with findings involving typically developing adolescents wherein physiological vulnerabilities (e.g., changes in circadian phases), environmental factors (e.g., increased internet use, responsibilities at school and work), schedule changes (e.g., early school start times), and increased autonomy (e.g., freedom to choose their own bedtime and bedtime routine) appear to increase risk for sleep disturbance (Loring, Johnston, Gray, Goldman, \& Malow, 2016). These risk factors can be exacerbated among individuals with ASD whereby deficits in executive functioning may inhibit the organization and regulation of sleep-conducive bedtime behavior (e.g., following a consistent bedtime routine, limiting caffeine consumption, restricting screen use at night; Quist, Chaplin \& Hendey, 2015). In addition, preliminary research suggests adolescents and young adults with ASD are more likely to have dysregulated levels of melatonin compared with typically developing controls (Tordjman et al., 2012).

Clinically significant sleep problems are detrimental to the overall wellbeing of individuals and their families. Specifically, insufficient sleep has been linked to increased severity of autism symptomatology, challenging behavior, and poor psychological wellbeing, as well as compromising parental sleep, quality of life, and marital relationships (Cortesi, Giannotti, Ivanenko, \& Johnson, 2010; Herrman, 2016). Without effective intervention, sleep problems experienced by adolescents with ASD may persist over time, compromising future functioning across home, school, and work settings. 
Behavioral intervention and parent-education programs have been effective in treating sleep disturbance in children with ASD (Cuomo et al., 2017). Increasingly, these interventions are informed by Functional Behavior Assessment (FBA), whereby an FBA is used to identify the variables influencing sleep problems and inform selection of individualized, multi-component, function-based treatments (e.g., Jin, Hanley, \& Beaulieu, 2013; McLay, France, Knight, Blampied, \& Hastie, 2019). Although there is evidence to support the use of function-based treatments for sleep problems in young children with ASD, there is little research on the utility of behavioral sleep interventions for adolescents with ASD. Further, FBA-informed sleep intervention has not been investigated with adolescents with ASD. Instead, pharmacological approaches, in particular melatonin, have been the most thoroughly researched treatment (Cuomo et al., 2017).

Most existing sleep-intervention research with typically developing adolescents involves the young person as the primary change agent. Specifically, involving young people in the sleep intervention increases their knowledge of sleep-conducive behavior and teaches them skills to resolve their own sleep disturbance (Schlarb, Liddle, \& Hautzinger, 2011). A review of the autism and sleep literature revealed that only one study has actively included adolescents with ASD in the therapeutic process. Loring et al. (2016) provided two sleepeducation sessions to 18 adolescents (11 to 18 years old) with high-functioning autism (HFA, IQ > 70) and their parents. Session one targeted sleep hygiene including bedtime routine and arranging the sleep environment, and session two taught relaxation and distraction techniques to facilitate sleep onset. Subsequent application of these practices by the adolescents, with parental support, resulted in significant improvements in sleep onset and efficiency. In other studies, young people with ASD have been engaged in the sleep-intervention process through social stories and visual schedules (e.g., Delemere \&Dounavi, 2018; Moore, 2004). However, in those cases, parents were the primary intervention agent.

When modifications are made to standard therapies to facilitate the engagement of adolescents with ASD, it is important to assess the social validity and acceptability of the procedures and include input from the adolescents (Callahan, Shukla Mehta, Magee, \& Wie, 2010). Unfortunately, most social validity data have been collected via parent report, even though parents did not directly experience the targeted sleep disturbance or treatment. Further, reliability between parent-reported sleep diaries and objective sleep measures (e.g., videosomnography) has predominantly been assessed with younger pre-adolescent children. Parents may be less likely to detect an adolescent's covert sleep-interfering behavior (e.g., electronic device use), and the validity of parent-reported sleep outcomes should not be assumed. 
A number of questions remain about the engagement of adolescents with ASD and sleep disturbance in their own treatment. First, the practicalities of including adolescents with ASD, across a range of intellectual functioning, in the assessment and treatment process are not well established. Second, the long-term effectiveness of behavioral sleep interventions for adolescents with ASD is understudied; specifically, there appears to be no study with follow up beyond 12 months (Durand, 2002; Weiskop, Matthews, \& Richdale, 2001). Third, little is known regarding adolescent perspectives and social validity of behavioral sleep interventions, which would seem particularly important when interventions include components delivered directly to the participant. Finally, although parent-reported sleep diaries are considered reliable among preschool and school-aged children (Hodge, Parnell, Hoffman, \& Sweeney, 2012), the reliability of parentreported sleep in adolescents with ASD warrants consideration. The present study evaluates (a) outcomes of individualized behavioral sleep interventions involving input from adolescents with ASD; (b) long-term maintenance of effects; (c) social validity of treatment components implemented with adolescents for sleep disturbance; and (d) the reliability and validity of parent-reported sleep diaries for adolescents with ASD.

\section{Method}

\section{Participants}

Participants ranged in age from 9 to 14 years old; although one participant was pre-adolescent, participants will be referred to as adolescents hereafter. Participants were referred to the research study by their parents or by professionals delivering services to individuals with ASD and their families. Each participant met the following inclusion criteria: (a) formal diagnosis of ASD or Asperger’s syndrome, as verified by a psychiatrist, registered psychologist, or pediatrician, and supported by results of the Gilliam Autism Rating Scale, Third Edition (GARS-3, Gilliam, 2013); (b) parent-reported difficulty initiating and maintaining sleep, supported by systematic in-home measurement; (c) no medical condition that directly interfered with sleep; and (d) sufficient receptive and expressive communication skills to engage in treatment. The communication criterion was assessed through clinical judgment coupled with item responses on the Communication domain of the Vineland Adaptive Behavior Scales Second Edition, Caregiver Rating Form (VABS-II; Sparrow, Cicchetti \& Balla, 2005). For example, the Vineland items considered included, "Follows instructions with one action and one object” and "Says at least 50 recognizable words”. Participant characteristics are summarized in Table 1 (pseudonyms have been used in place of real names to protect participant privacy).

\section{Procedures}


Experimental design. A single-case design, incorporating baseline [A], intervention [B], and shortand long-term follow-up phases, was used to evaluate treatment effects. Additional intervention phases were indicated by a phase-change line and alphabetization (C, D, or E). An AB design was applied to Niko and Eric, and an ABCDE design to Peter.

Setting. Participants were located throughout New Zealand. Clinical interviews and treatment planning discussions with families were conducted in a university-based clinic or at the participant's home if they were unable to travel to the clinic. The VABS-II and GARS-3 were administered to caregivers over the phone prior to the clinical interview. Other pre-treatment psychometric assessments were given to the parent at the clinical interview to complete and return to the researcher. Post-treatment questionnaires were sent to families upon conclusion of the intervention phase. Treatment was implemented within the participant's home by adolescents and their parents with the support of the first author. During treatment, communication with participants and families was conducted in person or via Skype, telephone, and email contact, depending on geographical location and participant preference.

Functional behavioral assessment. A combination of the Sleep Assessment Treatment Tool (SATT; Hanley, 2005), Questions About Behavioral Function (QABF; Matson \& Vollmer, 1995), sleep diaries, and analysis of video footage was used to conduct the FBA. The SATT is an open-ended interview tool designed to identify factors contributing to children's sleep disturbance; it was used to guide questions in the clinical interview. The QABF, a brief functional assessment checklist used to establish the function of a target behavior, was completed by parents following the clinical interview. Information gathered about the history and type of sleep problems, sleep hygiene practices, antecedent and consequence variables maintaining the sleep problem, and its possible function was synthesized in an FBA-informed case conceptualization (Blampied, 2013).

Baseline. Baseline commenced following completion of the FBA. Baseline length was staggered such that Peter, Niko, and Eric completed 3, 5, and 8 weeks of baseline recording respectively. Baseline length was determined by random assignment, though on occasion this was extended due to participant readiness to commence intervention (i.e., to ensure that the conclusion of baseline was commensurate with the beginning of treatment, baseline was occasionally extended). During baseline, families were asked to continue with their existing sleep practices (e.g., bedtime routine, electronic device use).

Intervention. Individualized, FBA-based, multi-component interventions commenced upon conclusion of baseline. The chosen treatment was discussed with parents and participants using the guided participation 
model to ensure a shared understanding of the issues and intervention (Sanders \& Burke, 2014). Treatment included components implemented with both parent and participant. Each treatment component was selected to address hypothesized factors underlying participant sleep disturbance, facilitate treatment compliance, and support the maintenance of helpful sleep habits. Table 2 includes a summary of each participant's sleep problem, FBA data, and subsequent intervention components.

During the intervention phase, researchers communicated daily with parents. Daily (Niko) and weekly (Peter and Eric) contact was also maintained with adolescent participants. During regularly scheduled contact, researchers provided participants with feedback regarding treatment implementation, treatment fidelity was monitored, and praise and encouragement was given. The intervention continued until the participant's sleep disturbance had been significantly reduced or eliminated, and this pattern had been evident consistently across a 10-to-14-day period. The intervention phase lasted for 48, 94, and 84 nights respectively for Niko, Peter, and Eric. FBA results and individualized treatments are detailed below (additional details regarding interventions are available from the first author).

The FBA revealed numerous antecedent and consequence variables appeared to be interfering with participants' sleep. These included lack of physiological sleep pressure due to inconsistent sleep/wake times and daytime sleep; stimulating activities pre-bedtime; presence of intrusive internal stimuli (e.g., reports of distressing cognitions); inappropriate sleep dependencies (e.g., electronic devices); lack of discriminative stimuli for bedtime (e.g., inconsistent bedtime routines); sleep environment discomfort; and exposure to light. Reinforcement contingencies for sleep-interfering behavior came in the form of parental attention; access to electronic devices and other preferred items (e.g., food and drink); and purported escape from intrusive internal stimuli (reduced distress).

Intervention components implemented with all three participants included discussions about key sleepfacilitative behaviors (e.g., closing eyes), the impact of sleep disturbance on areas of importance to them (e.g., ability to play video games well), and connection between their behavior and sleep disturbance. All three also received instruction in relaxation training (e.g., progressive muscle relaxation [PMR], deep breathing) which has been suggested to reduce physiological arousal and support independent sleep onset (Stewart \& Gordon, 2014). All parents received psychoeducation regarding the relationship between existing operant and respondent conditioning processes and their child's sleep disturbance, sleep hygiene (Jan et al., 2008), modified extinction (Kuhn \& Weidinger, 2000), and positive reinforcement strategies. 
Varying topographies and functions of sleep problems necessitated the use of additional individualized intervention components. Individualized components implemented included (a) social stories to depict new targeted sleep routines, sleep-conducive behavior, and reinforcement contingencies (Gray \& Garand, 1993); (b) sleep checklists (a visual schedule of the bedtime routine); (c) visualization techniques to re-direct sleepinterfering cognitions; and (d) Gro-clocks (to provide a discriminative stimulus for sleep/wake times). Verbal instruction, social stories, modeling, visual aids (e.g., picture cues), parent presence, and participant interests were integrated to facilitate participants’ comprehension and engagement with therapeutic resources. Individualized parent-mediated interventions included (a) bedtime fading and sleep restriction (Vriend, Corkum, Moon, \& Smith, 2011) to increase physiological sleep pressure and create a motivating operation for sleep; (b) appropriate sleep dependencies (provision of sleep-conducive stimuli that were accessible throughout the night e.g., a soft toy; Jin et al., 2013); (c) clear discriminative stimuli for bed preparation and sleep onset (e.g., consistent statements about bedtime and sleep); (d) scheduled access to putative reinforcers (Jin et al., 2013); (e) graduated extinction (Vriend et al., 2011); and (f) positive reinforcement for successive approximation towards desired sleep behavior.

Niko. Niko’s primary sleep problems included frequent and prolonged night-wakings (NWs) as well as early wakings (EWs, i.e., any rise time before 6:00am where sleep was not reinitiated). FBA results suggested Niko’s sleep disturbance was maintained by access to tangible reinforcement (electronic device use) and escape from the perceived discomfort and cold of his bed to a heated lounge. Lack of physiological sleep pressure due to daytime sleep was also suggested to be contributing to Niko’s sleep difficulties.

The following treatment components were implemented simultaneously: sleep hygiene; psychoeducation; a social story; a comfortable sleep environment; bedtime fading and sleep restriction; relaxation techniques; modified extinction (restricted access to electronic devices and lounge heater); Gro-clock; and positive reinforcement for successive approximation towards desired sleep behavior (e.g., remaining in bed until successively later from 5:00am). The preceding techniques functioned to increase sleep pressure, reduce reinforcement for sleep-interfering behavior, and promote engagement in sleep-conducive behavior. Niko showed a reluctance to stop using electronic devices during the night, despite psychoeducation. As access to electronic devices was hypothesized to be the primary reinforcer of sleep-interfering behavior, restricted access was imperative. Niko was taught relaxation techniques, as a replacement behavior for leaving his bedroom when he woke, to facilitate re-initiation of sleep. Niko agreed a Gro-clock was necessary to signal appropriate sleep/wake times as he was unable to read the time, enabling him to earn rewards. Immediate reinforcement for 
improvements in sleep outcomes was provided to strengthen Niko's participation in therapy, as his parent indicated he would become unmotivated and non-compliant rapidly without immediate reinforcement for progress. Reinforcement options were collaboratively agreed on by Niko and his parent.

Peter. Peter's primary sleep problems included delayed sleep-onset latency (SOL) and EWs thought to be reinforced by access to preferred items (e.g., electronic devices). Additional precipitating and maintaining factors included lack of physiological sleep pressure, inappropriate sleep dependencies (e.g., electronic device use), and lack of discriminative stimuli for bedtime. Intervention phase one consisted of teaching Peter relaxation strategies and providing a social story. These were implemented first to provide a rationale for and prepare Peter for later changes, provide him with skills to manage anxiety, and facilitate sleep onset. These components also reduced parent anxiety regarding Peter's capacity to cope with change and thereby enhanced treatment fidelity.

Phase two of intervention included consistent implementation of appropriate sleep dependencies (e.g., soft toy); discriminative stimuli for sleep (e.g., switching off the bedroom light at bedtime); and gradual extinction of mobile phone use, achieved by scheduled access to all of his devices until 15 min prior to bedtime at which point he was asked to place his mobile phone in a visually enticing "Finished Box", and was reinforced for compliance (immediately provided an edible treat e.g., chocolate coin). Peter chose a sleep item (e.g., soft toy) to take to bed with him, as an appropriate sleep dependency, and his parents issued a consistent sleep statement and turned off his bedroom light. Peter's bright nightlight was replaced with a dim one to facilitate melatonin secretion and reduce the visibility of preferred items, while continuing to provide a source of comfort. Bedtime fading and elimination of daytime sleep was an additional component implemented to ensure sufficient physiological sleep pressure.

Intervention phases three and four included the above components plus the gradual extinction of Peter's iPad and then laptop respectively. Reinforcement for compliance was faded as Peter learnt to independently put his devices away and go to bed on time. Phase five consisted of fading Peter's bedtime earlier in 15-min increments to an age appropriate time.

Eric. Eric’s primary sleep problems included frequent curtain calls (CCs, i.e., bids for parental attention) and a delayed SOL. His FBA revealed his sleep disturbance was maintained by both positive and negative reinforcement contingencies, including access to tangibles (electronic devices and food), social attention, and by sleep-interfering cognitions (e.g., “What if something happens to Mom when I’m asleep?”). 
Antecedent variables implicated in Eric’s sleep disturbance included lack of physiological sleep pressure, hyperarousal, and exposure to bright light and stimulating electronic device content prior to bed. Exposure to bright light from electronic devices immediately before bedtime may have interfered with Eric’s natural melatonin secretion. Furthermore, exposure to device content was suggested to interfere with Eric's ability to reach a relaxed state.

Intervention included simultaneous implementation of psychoeducation (instruction regarding the importance of sleep, and the impact of sleep-interfering and sleep-conducive behavior); a sleep checklist; restricted access to electronic devices prior to bedtime; modified extinction (minimal parent response to CCs); bedtime fading and sleep restriction; and relaxation and visualization (taught to picture a pleasant/peaceful scene during sleep onset). Following consultation with the researcher, Eric agreed to stop using electronic devices after dinner and further enforcement of restrictions was not necessary. Eric’s sleep checklist supported his tendency for rule-following and bedtime routine compliance. Collaboration regarding checklist items provided Eric some control over his bedtime routine and was intended to increase his motivation to adhere to intervention. Giving him a relaxing bedtime routine, relaxation instruction, and sleep restriction functioned to reduce the hypothesized association between bed and hyperarousal. Eric’s mother was also instructed to minimize interactions post-bedtime and Eric was taught relaxation skills to facilitate independent management of sleep-interfering cognitions and reduce reinforcement for sleep-interfering behavior.

Short- and long-term follow-up. Short- and long-term follow up data were collected for 1 week using sleep diaries at 3 to 5 weeks and 12 to 13 weeks post-treatment. An 18-to-24-month follow-up phone interview was conducted with parents at which time they were also asked to begin a 7-day sleep diary. However, only Eric’s family completed the extended follow up diary.

\section{Measures}

Clinical interviews. Separate clinical interviews were conducted with each family and participant prior to commencing baseline. Interviews were supplemented with visual aids to support communication. Information on past and present sleep disturbance, participant developmental history, family context, and possible environmental (e.g., bedtime routine) and mental health factors (e.g., anxiety) that could be interfering with sleep were discussed. 
Parent-reported sleep diaries. Parents recorded data in daily sleep diaries during each phase of the study. Diaries were used to record (a) frequency and duration of daytime sleep; (b) duration of SOL; (c) frequency of CCs; (d) frequency and duration of NWs; and (e) time of morning waking. The latter was used to calculate discrepancy between actual and goal wake time and identify incidents of EW. Participants’ sleep setting, behavior during CCs and NWs, as well as parents' responses to this behavior were also noted. Sleep diaries were returned to the research team on a weekly basis.

Videosomnography. Swann Advanced-Series DVR4-1200, nighttime, infrared video cameras were used to record participant's sleep and to permit the coding of interobserver agreement (IOA) data. Information obtained from video included (a) topographies of awake behavior post-bedtime (e.g., vocalizations, stereotypy, play); (b) topographies of sleep behavior (e.g., sleep position, eye movement, limb movement); (c) duration of SOL; (d) frequency of CCs; (e) frequency and duration of NWs; and (f) time of morning waking. The following operational definitions were used to code video (a) asleep, lying down with minimal nondiscrete movement and no indication of wakefulness; and (b) awake, the presence of any sleep-interfering behavior, eyes open, or frequent physical movement (Jin et al., 2013). Recording began when the participant went to bed and ended when they awoke to begin the day. Video footage was downloaded to an external hard drive and distributed regularly to the research team to enable objective monitoring of participant progress.

Child Sleep Habits Questionnaire (Owens, Spirito, \& McGuinn, 2000). The Child Sleep Habits Questionnaire (CSHQ) was completed during assessment and post-treatment to evaluate change in parentreported sleep disturbance. The CSHQ was completed by Niko and Eric's parents. Peter's parents did not complete the CSHQ as Peter was not within the measure's validated age range. The CSHQ is a parent-report questionnaire consisting of 45 items relating to children's sleep patterns, scores $>41$ are indicative of clinically significant sleep disturbance (Owens et al., 2000).

Child Behavior Checklist for Ages 6-18 (Achenbach, 2001). The Child Behavior Checklist (CBCL) is a 113-item parent report measure of internalizing (e.g., withdrawn) and externalizing (e.g., aggressive) behavior. The CBCL was completed by parents during assessment and used to indicate the extent of additional behavioral difficulties experienced by participants.

Gilliam Autism Rating Scale. The GARS-3 was used to corroborate ASD diagnoses and indicate symptom severity. The GARS-3 is a 56-item informant rating scale of Autism symptomatology (Gilliam, 2013). It is designed to assess the likelihood a person has ASD and the severity of their behavior in accordance with the 
Diagnostic and Statistical Manual of Mental Disorders (DSM-5). Items are summed to provide a total ASD Index score. Higher scores indicate a high likelihood and increased severity of ASD.

Treatment acceptability. The Treatment Acceptability Rating Form-Revised (TARF-R; Reimers, Wacker, Cooper, \& DeRaad, 1992) was administered post-treatment to assess parents’ perceptions of overall treatment acceptability. The TARF-R consists of 17 items which examine ratings of treatment acceptability and three items assessing problem severity and participants’ understanding of the intervention approach. Ratings on six subscales (Effectiveness; Reasonableness; Willingness; Cost; Negative side-effects; Disruption/time) are summed to provide a total treatment acceptability score. In addition, parents and their child were interviewed separately to assess the social validity of treatment and to provide qualitative information regarding treatment effects. Information pertaining to sleep (e.g., fatigue, sleep quality), secondary outcomes (e.g., mood), preferred and non-preferred assessment and treatment components, knowledge regarding healthy sleep habits, and suggestions for improvement were gathered. The format of the participant interview included open questions, closed questions with multiple choice options, and visual aids (e.g., photos of treatment components) to facilitate communication.

Interobserver agreement (see Table 3). Video footage was coded by a researcher blind to parent sleep diary recordings. Agreement between parent report and direct observation data extracted from video was then calculated. Sleep phenomena which parents could not be expected to detect (e.g., covert awakenings in which the adolescent remained quiet in their bed) were omitted from IOA calculations. Measures of duration (e.g., SOL), sleep, and wake times were considered in agreement if parent and video were \pm 15 min. Percent agreement for each behavior was calculated using the equation [Agreement/ (Agreement + Disagreement)] $\times$ $100 \%$. IOA data were collected for $22 \%, 46 \%$, and $5 \%$ of nights across baseline and treatment phases for Niko, Peter, and Eric respectively. Incomplete or lack of sleep diary entries on nights when video data was available, inhibited calculation of IOA on more nights. Limited IOA data were collected for Eric as he withdrew consent for video recording on night 107 due to feeling it was an invasion of his privacy. Niko’s mean IOA was 86\% for duration of NWs and 100\% for duration of EWs. Peter's mean IOA was 93\% (range, 85-97\%) for SOL and 98\% (range, 86-100\%) for duration of EWs. Eric’s mean IOA was 95\% (range, 91-100\%) for CCs and 100\% for SOL.

Treatment fidelity (see Table 4). A checklist based on a task analysis of each measurable parentmediated treatment component (e.g., consistent bedtime) was created for all families. Parent treatment fidelity 
was assessed by comparing the first author's daily contact notes, video footage, and sleep diaries with the protocol outlined in the treatment checklist. Parent treatment fidelity was calculated for $90 \%$ or more of intervention nights across all participants, using the formula (Completed tasks/ Total tasks) $\times 100 \%$. An aggregate score was then calculated for each participant. Treatment fidelity scores were 93\%, 98\%, and 71\% for Niko, Peter, and Eric respectively (mean = 87\%). Niko, Peter, and Eric's parents followed every component of the treatment plan (i.e., reached $100 \%$ treatment fidelity) on $72 \%, 84 \%$, and $26 \%$ of nights respectively.

Treatment fidelity for each adolescent participant was assessed by examining participant report and video for evidence of treatment adherence. The imperceptible nature of some of the components (e.g., visualization) and unreliable reporting inhibited direct measurement of treatment fidelity; however, there was indirect evidence of intervention compliance by adolescents with ASD. Niko demonstrated mastery of relaxation skills during intervention sessions and was able to identify when to utilize such strategies. However, he was rarely observed to apply these within the sleep context. There was little video evidence of Niko or Peter completing relaxation exercises. After Niko's access to electronic devices was restricted via password protection he complied with the treatment plan and remained in bed, stating he closed his eyes to reinitiate sleep upon waking as opposed to utilizing relaxation strategies. Initial video footage of Eric revealed he completed PMR frequently prior to sleep onset. Eric reported using his sleep checklist and deep breathing strategies every night, although he noted that he did not always stick consistently to his bedtime routine during school holidays.

\section{Data Analyses}

Visual analyses. Visual analysis was used to assess the effectiveness of FBA-informed interventions and additional therapies. Level, variability, and trend in the data were evaluated across study phases (Kazdin, 2001).

Effect size estimate. Percentage below the median (PBM) is the percentage of intervention data points below the baseline median (calculated thus because the behavior is decreasing; Parker, Vannest \& Davis, 2011). For example, if the baseline median was 100 and 25/25 intervention data points were below 100 (PBM = 100\%), whereby if 50\% of the data points were below $100(\mathrm{PBM}=50 \%)$. Visual analysis was supplemented by calculating PBM to estimate effect sizes: $<70 \%$ represents ineffective treatment; 70 to $90 \%$ moderate effectiveness; and >90\% high effectiveness (Ma, 2009). The reliable change index (RCI) was used to ascertain whether differences in pre-and post-CSHQ scores reflected true significant change as opposed to measurement 
error (Jacobson \& Truax, 1991), clinical change occurred when pre CSHQ scores reduced from the clinical range to the normal range.

\section{Results}

Treatment results for each participant are presented individually in a case study-format. Sleep diary data for each participant are presented in Figs. 1, 2, and 3.

\section{Data quality}

Video footage was recorded on 20-88\% of nights across all participants throughout baseline and treatment phases. Parents recorded sleep diary data for all dependent variables across baseline and treatment on 37-46\% of nights (i.e., although parents filled out sleep diaries regularly, they did not collect information on each variable each night).

Niko’s baseline sleep diary data is scarce as his parent had difficulty recording diary data during that phase, this prevented calculation of IOA in baseline. There are no sleep diary data for Peter from nights 80 to 102; Peter's parents recorded video only on these nights. Eric's SOL sleep diary data is scarce from night 36 to 85 as school holidays resulted in variability in sleep settings (e.g., tent, friend's house).

Niko

Niko displayed high variability in the duration of NWs (0 to $120 \mathrm{~min}$ ) and EWs (0 to $180 \mathrm{~min}$ ) in baseline (Fig. 1). There was an immediate reduction in the level and variability of both sleep variables, with PBM scores $(\mathrm{NW}=100 \%, \mathrm{EW}=95 \%)$ demonstrating a large treatment effect. NWs and EWs were eliminated by the end of treatment and these effects were maintained at both short- and long-term follow up.

At the 24-month follow-up, Niko and his parent reported that the elimination of NWs and EWs had been maintained. Access to devices was still restricted during the night, and Niko used a watch as opposed to a Gro-clock to signal an appropriate rise time. Additional treatment techniques were no longer required.

\section{Peter}

Peter's SOL was highly variable during baseline (range = 5 to $195 \mathrm{~min}$; see Fig. 2). There was an immediate reduction in the level of SOL upon implementation of relaxation strategies. The level and variability reduced further from intervention phases two to four. A large treatment effect was observed within intervention phase four $(\mathrm{PBM}=100 \%)$. SOL treatment effects were maintained at follow up. The duration of Peter's EWs 
was highly variable during baseline (range 0 to $180 \mathrm{~min}$ ). There was an immediate reduction in the level and variability of the duration of EWs at treatment onset and they were eliminated during treatment, with this result maintained at short- and long-term follow up.

At the 18-month follow-up parents indicated that Peter was not experiencing sleep disturbance, SOL was 15 min, and he was not experiencing NWs or EWs. Peter's family still used the Finished Box and restricted his sleep during the day. The social story, sleep item, and reward system were no longer required.

Eric

In baseline Eric displayed a high frequency of CCs and significant variability (range = 0 to 6; see Fig. 3). There was an immediate reduction in the level and variability of CCs with intervention. Treatment had a moderate effect on CCs (PBM = 88.5\%). The high frequency of Eric's CCs on nights 107 and 114 occurred during his transition to a new school. SOL was prolonged and highly variable during baseline (range $=15$ to 450 min) but reduced significantly during intervention $(\mathrm{PBM}=100 \%)$, and this was maintained at short- and longterm follow-up.

At 18-month follow-up, Eric’s parent reported Eric was not experiencing sleep problems and no longer engaged in bedtime resistance. Eric’s mother said his SOL was 10 to 15 min in duration. The family reported continued use of delayed bedtime, restricted access to devices, and use of deep breathing.

\section{CSHQ Scores}

Both Niko and Eric experienced a reliable change in their CSHQ scores (see Table 5). For Eric this change was clinically significant, with his post-treatment score falling below the clinical cut-off for sleep disturbance.

\section{Social Validity}

Participant reports suggest FBA-informed sleep interventions and components implemented with adolescents were acceptable to participants and their parents. Planned use of tangible and social rewards was well regarded. Participants varied in their rating of relaxation. Eric reported finding deep breathing helpful. He attributed his success to this and noted that engaging in deep breathing at bedtime helped prevent his mind from "buzzing” as he focused on counting his inhalations and exhalations. Niko and Eric reported PMR was ineffective and Peter was ambivalent. Peter was the only participant to indicate he did not like the elimination of 
electronic device use. Some participants commented on the intrusive nature of the video camera and the discomfort and time-consuming nature of having to talk to a researcher daily. Niko and Eric indicated they had experienced improvements in their sleep, including reduced SOL and daytime fatigue, as well as ability to reinitiate sleep upon waking.

During post-treatment interviews all parents reported that the intervention had successfully reduced their child’s sleep disturbance and perceived daytime fatigue. Importantly, parents felt their child had developed the skills to manage their sleep independently by implementing “the tools in his strategy bag” (e.g., deep breathing) and adhering to sleep-facilitative behavior (e.g., remaining in bed). Peter's parents reported that, while visual aids were important for his comprehension, they also facilitated parental structure and routine. Eric's treatment, which consisted predominantly of intervention components delivered directly to him, was described by his parent as “non-invasive”.

Treatment Acceptability Rating Form- Revised. TARF-R scores have a possible range of 17 to 119; higher scores indicate higher acceptability. Parent ratings ranged from 94 to 113 (see Table 6). Each parent’s rating yielded the maximum score on the Effectiveness subscale. Overall, parents rated the interventions to be highly acceptable, effective, and easy to understand, taking little time to implement, at no financial cost.

\section{Discussion}

The purpose of this study was to evaluate the effects of individualized behavioral sleep interventions involving input from adolescents with ASD alongside parent-mediated treatment with a focus on maintenance and social validity. Further, the validity of parent-report sleep diaries was also assessed. Overall, results of this pilot study suggest that comprehensive, individualized interventions including both adolescent- and parentimplemented treatment components can reduce sleep disturbance. Parents reported that improvements in sleep were maintained at 18 and 24 months post-intervention. However, caution is warranted in interpreting these maintenance data because of reliance on parent report and lack of direct observation. Overall, the treatment components were rated as acceptable (socially valid) by adolescents with ASD and their parents. Specifically, all participants reported that they enjoyed the reinforcement systems and two reported that the adolescentimplemented treatment components (e.g., deep breathing) were beneficial.

Successful application of intervention components delivered directly to the adolescent appeared to have been mediated in part, by each participant’s functioning and their use of individualized treatment strategies (e.g., relaxation). Sleep problems resolved to the greatest extent for Eric who had the highest communicative abilities 
and participant treatment fidelity. By contrast, Niko and Peter required additional reinforcement contingencies to increase sleep-conducive behavior. Individuals with more severe intellectual disability may struggle to refrain from engaging in sleep-interfering behaviors that offer immediate reinforcement and may experience difficulty generating appropriate alternative responses (Ho, Stephenson, \& Carter, 2015). Niko attempted to gain access to electronic devices as intervention began, only engaging in sleep facilitative behavior after access had been restricted. Although Eric evidenced relaxation training mastery, Peter required significant support to learn relaxation skills (e.g., social story, visual aids, modeling). After four sessions, his relaxation technique still did not appear correct and he was not able to identify when to utilize the strategies. Echolalia and compromised memory and sequencing abilities appeared to limit Peter’s ability to engage in conversation. Niko and Peter also each had difficulty attending to therapeutic tasks. Adolescents with ASD are less likely to initiate social interaction (Chevallier Kohls, Troiani, Brodkin, \& Schultz, 2012). Limited social skills may have contributed to the inhibited engagement with Peter and Niko.

Parents within the current study consistently reported that adolescents had become responsible for their own sleep and had developed skills to engage in appropriate sleep behavior independently, likely facilitating the maintenance of treatment effects over an extended time period. Psychoeducation may have been sufficient for Eric to reduce use of electronic devices without resistance, particularly as this was not the primary maintaining reinforcer for his sleep-interfering behavior. Furthermore, an increase in Peter’s sleep-conducive behavior (e.g., eyes closed, lying still) was observed following explicit, concrete instructions within a social story. Interestingly, he also began reprimanding other family members for sleeping during the day. Parents play an important role enforcing limits to facilitate sleep-conducive behavior and delivering treatment directly to adolescents provides them with the skills and knowledge to sustain healthy sleep practices. Treatment maintenance is critical to help prepare adolescents with ASD for adulthood and ensure they can function most effectively within living, education, and vocational settings without being compromised by the effects of sleep disturbance.

Parent treatment fidelity was an issue for one of the three families. Eric's parent completed every component of the treatment plan on $26 \%$ of nights. It was particularly difficult for this family to refrain from providing social attention post-bedtime and to maintain consistent sleep and wake times respectively. In this case, low parent treatment fidelity did not appear to affect Eric’s sleep outcomes, perhaps because his own treatment fidelity was high. Treatment fidelity was not a challenge for Niko and Peter's parents. The complexity of everyday life for parents of children with neurodevelopmental disorders may impact their ability to 
consistently focus their attention and energy on improving their child's sleep and prioritize this one aspect of their lives (Beresford, Stuttard, Clarke, \& Maddison, 2016).

Videosomnography has rarely been used within the ASD and sleep literature; however, this objective measure can be collected within participants’ homes and enables detection of salient information (e.g., topographies of sleep and awake behavior) unobtainable through actigraph or polysomnography recordings (Moore, Evans, Hanvey, \& Johnson, 2017). High IOA between parent-reported sleep diaries and videosomnography was found, replicating results of previous sleep research with young people with ASD (Jin et al., 2013). However, our videosomnography data revealed parents were often unable to detect covert sleepinterfering behavior (e.g., early morning device use). As a result, they struggled to identify the length of sleep onset or wakings. Subjective sleep measures may be more reliable within pre-school and school- aged populations, as sleep-interfering behavior tends to involve overt signaling to the parent or significant disruption to the household. Understandably, as Eric's case suggests, increasing desire for privacy may prevent adolescent participants consenting to the use of videosomnography. Further, increasing sexual desire during adolescence, paired with the lack of social understanding apparent in many individuals with ASD, may put this population at risk of being inadvertently recorded while engaging in sexual activities. Privacy issues necessitate careful consideration of videosomnography with any population, but particularly perhaps with adolescents.

\section{Limitations and Future Directions}

The current study illustrates treatment components directed towards adolescents are viable and may be beneficial additions to traditional parent-mediated sleep interventions when working with verbal adolescents with ASD. Future research aimed at determining the extent to which intellectual functioning and motivation may influence the effectiveness of sleep interventions implemented with adolescents appears warranted. Of consideration within the current study is the small number of participants with heterogeneous presentations of sleep problems and ASD characteristics, inhibiting generalizability to other adolescents with ASD. Second, recording covert sleep-interfering behavior via parent report may not be sufficiently accurate, suggesting a need for research aimed at evaluating the veracity of dependent variables used in sleep-intervention research. Third, the single-case design used did not isolate the effects of any specific intervention component and it is not possible to determine whether any given component was necessary or sufficient. However, the results of this pilot study suggest future research could include a component analysis and experimental design capable of demonstrating experimental control. 


\section{Author Contributions:}

JvD: designed and executed the study, conducted data analyses, and wrote the paper. LM and KF contributed equally towards the collaboration of the design, execution and writing of the study. NB: collaborated with the data analysis and writing of the study. RL: collaborated with the writing of the study. $\mathrm{JH}$ : assisted with clinical application of assessment and intervention to Peter.

\section{Funding Information:}

This study was partially supported by the Health Research Council of New Zealand (grant number 17/852).

\section{Compliance with Ethical Standards:}

Conflict of Interest: The authors declare that they have no conflict of interest.

Ethical approval: All procedures performed in studies involving human participants were in accordance with the ethical standards of the University of Canterbury Human Ethics Committee and with the 1964 Helsinki declaration and its later amendments or comparable ethical standards.

Informed consent: Informed consent was obtained from all participants. 


\section{References}

Achenbach, T. (2001). Child Behavior Checklist for Ages 6-18. Burlington, VT: ASEBA.

Baker, E., Richdale, A., Short, M., \& Gradisar, M. (2013). An investigation of sleep patterns in adolescents with high-functioning autism spectrum disorder compared with typically developing adolescents. Developmental Neurorehabilitation, 16, 155-165. doi:10.3109/17518423.2013.765518.

Beresford, B., Stuttard, L., Clarke, S., \& Maddison, J. (2016). Parents’ experiences of psychoeducational sleep management interventions: A qualitative study of parents of children with neurodevelopmental disabilities. Clinical Practice in Pediatric Psychology, 4, 164-175. doi:10.1037/cpp0000144.

Blampied, N. M. (2013). Functional behavioral analysis of sleep in infants and children. In A. R. Wolfson \& H.E. Montgomery-Downs (Eds.), The Oxford handbook of infant, child, and adolescent sleep and behavior (pp. 169-188). New York, NY: Oxford University Press.

Callahan, K., Shukla-Mehta, S., Magee, S., \& Wie, M. (2010). ABA versus TEACCH: The case for defining and validating comprehensive treatment models in autism. Journal of Autism and Developmental Disorders, 40, 74-88. doi:10.1007/s10803-009-0834-0.

Chevallier, C., Kohls, G., Troiani, V., Brodkin, E. S., \& Schultz, R. T. (2012). The social motivation theory of autism. Trends in Cognitive Sciences, 16, 231-239. doi:10.1016/j.tics.2012.02.007.

Cortesi, F., Giannotti, F., Ivanenko, A., \& Johnson, K. (2010). Sleep in children with autistic spectrum disorder. Sleep Medicine, 11, 659-664. doi:10.1016/j.sleep.2010.01.010.

Cuomo, B. M., Vaz, S., Lee, E. A. L., Thompson, C., Rogerson, J. M., \& Falkmer, T. (2017). Effectiveness of Sleep-Based interventions for children with autism spectrum disorder: A Meta-Synthesis. Pharmacotherapy: The Journal of Human Pharmacology and Drug Therapy, 37, 555-578. doi:10.1002/phar.1920

Delemere, E., \& Dounavi, K. (2018). Parent-implemented bedtime fading and positive routines for children with autism spectrum disorders. Journal of Autism and Developmental Disorders, 48, 1002-1019. doi:10.1007/s10803-017-3398-4.

Durand, V. M. (2002). Treating sleep terrors in children with autism. Journal of Positive Behavior Interventions, 4, 66-72. 
Elrod, M. G., Nylund, C. M., Susi, A. L., Gorman, G. H., Hisle-Gorman, E., Rogers, D. J., \& Erdie-Lalena, C. (2016). Prevalence of diagnosed sleep disorders and related diagnostic and surgical procedures in children with autism spectrum disorders. Journal of Developmental and Behavioral Pediatrics, 37, 377-384. doi:10.1097/DBP.0000000000000248.

Gilliam, J.E. (2013). Gilliam autism rating scale-third edition. Austin TX: Pro-Ed.

Goldman, S. E., Richdale, A. L., Clemons, T., \& Malow, B. A. (2012). Parental sleep concerns in autism spectrum disorders: Variations from childhood to adolescence. Journal of Autism and Developmental Disorders, 42, 531-538. doi:10.1007/s10803-011-1270-5.

Gray, C. A., \& Garand, J. D. (1993). Social stories: Improving response of students with autism with accurate social information. Focus on Autistic Behavior, 8, 1-10.

Hanley, G. P. (2005). Sleep Assessment and Treatment Tool [Measurement Instrument]. Retrieved May 26, 2017, from https://practicalfunctionalassessment.files.wordpress.com/2015/06/satt.pdf.

Herrmann, S. (2016). Counting sheep: Sleep disorders in children with autism spectrum disorders. Journal of Pediatric Health Care, 30, 143-154. doi:10.1016/j.pedhc.2015.07.003.

Ho, B. P. V., Stephenson, J., \& Carter, M. (2015). Cognitive-behavioral approach for children with autism spectrum disorder: A literature review. Journal of Intellectual and Developmental Disability, 40, 213229. doi:10.3109/13668250.2015.1023181.

Hodge, D., Parnell, A. M. N., Hoffman, C. D., \& Sweeney, D. P. (2012). Methods for assessing sleep in children with autism spectrum disorders: A review. Research in Autism Spectrum Disorders, 6, 1337 1344. doi:10.1016/j.rasd.2012.05.009.

Jacobson, N. S., \& Truax, P. (1991). Clinical significance: A statistical approach to defining meaningful change in psychotherapy research. Journal of Consulting and Clinical Psychology, 59, 12-19. doi:10.1037//0022-006X.59.1.12.

Jan, J. E., Owens, J. A., Weiss, M. D., Johnson, K. P., Wasdell, M. B., Freeman, R. D., \& Ipsiroglu, O. S. (2008). Sleep hygiene for children with neurodevelopmental disabilities. Pediatrics, 122, 1343-1350. doi:10.1542/peds.2007-3308. 
Jin, C. S., Hanley, G. P., \& Beaulieu, L. (2013). An individualized and comprehensive approach to treating sleep problems in young children. Journal of Applied Behavior Analysis, 46, 161-180. doi:10.1002/jaba.16.

Kazdin, A, E. (2001). Behavior modification in applied settings (6th ed.). Belmont, CA: Wadsworth/Thompson Learning.

Kuhn, B. R., \& Weidinger, D. (2000). Interventions for infant and toddler sleep disturbance: A review. Child \& Family Behavior Therapy, 22, 33-50. doi:10.1300/J019v22n02_03.

Loring, W. A., Johnston, R., Gray, L., Goldman, S., \& Malow, B. (2016). A brief behavioral intervention for insomnia in adolescents with autism spectrum disorders. Clinical Practice in Pediatric Psychology, 4, 112-124. doi:10.1037/cpp0000141.

Ma, H. (2009). The effectiveness of intervention on the behavior of individuals with autism: A meta-analysis using percentage of data points exceeding the median of baseline phase (PEM). Behavior Modification, 33, 339-359. doi:10.1177/0145445509333173.

Malow, B. A., Katz, T., Reynolds, A. M., Shui, A., Carno, M., Connolly, H. V., . . Bennett, A. E. (2016). Sleep difficulties and medications in children with autism spectrum disorders: A registry study. Pediatrics, 137, 98-104. doi:10.1542/peds.2015-2851H.

Matson, J. L. \& Vollmer, T. R. (1995). User's guide: Questions About Behavioral Function (QABF). Baton Rouge, LA: Scientific Publishers.

McLay, L. K., France, K. G., Knight, J., Blampied, N. M., \& Hastie, B. (2019). The effectiveness of functionbased interventions to treat sleep problems, including unwanted co-sleeping, in children with autism. Behavioral Interventions, 34, 30-51. doi:10.1002/bin.1651.

Moore, M., Evans, V., Hanvey, G., \& Johnson, C. (2017). Assessment of sleep in children with autism spectrum disorder. Children, 4, 72. doi:10.3390/children4080072.

Moore, P. S. (2004). The use of social stories in a psychology service for children with learning disabilities: A case study of a sleep problem. British Journal of Learning Disabilities, 32, 133-138. doi:10.1111/j.1468-3156.2004.00278.x. 
Owens, J. A., Spirito, A. \& McGuinn, M. (2000). The Children’s Sleep Habits Questionnaire (CSHQ): Psychometric properties of a survey instrument for school-aged children. Sleep, 23, 1-9.

Parker, R. I., Vannest, K. J., \& Davis, J. L. (2011). Effect size in single-case research: A review of nine nonoverlap techniques. Behavior Modification, 35, 303-

\section{2. https://doi.org/10.1177/0145445511399147}

Quist, H., Chaplin, E., \& Hendey, O. (2015). Sleep intervention for adults with autism spectrum condition. Mental Health Practice, 18, 14-18. doi:10.7748/mhp.18.10.14.e937

Reimers, T. M., Wacker, D. P., Cooper, L. J., \& DeRaad, A. O. (1992). Clinical evaluation of the variables associated with treatment acceptability and their relation to compliance. Behavioral Disorders, 18, 6776.

Richdale, A. L., \& Schreck, K. A. (2009). Sleep problems in autism spectrum disorders: Prevalence, nature, \& possible biopsychosocial aetiologies. Sleep Medicine Reviews, 13, 403-411. doi:10.1016/j.smrv.2009.02.003

Sanders, M. R., \& Burke, K. (2014). The “Hidden” technology of effective parent consultation: A guided participation model for promoting change in families. Journal of Child and Family Studies, 23, 12891297. doi:10.1007/s10826-013-9827-x

Schlarb, A. A., Liddle, C. C., \& Hautzinger, M. (2011). JuSt - a multimodal program for treatment of insomnia in adolescents: A pilot study. Nature and Science of Sleep, 3, 13-20. doi:10.2147/NSS.S14493

Sparrow, S. S., Cicchetti, D. V. \& Balla, D. A. (2005). Vineland Adaptive Behavior Scales: Second Edition (Vineland II), Survey Interview Form/Caregiver Rating Form. Livonia, MN: Pearson Assessments.

Stewart, S. E., \& Gordon, J. E. (2014). Parent-assisted cognitive-behavioural therapy for children's nighttime fear. Behaviour Change, 31, 243-257. doi:10.1017/bec.2014.19

Tordjman, S., Anderson, G. M., Bellissant, E., Botbol, M., Charbuy, H., Camus, F., .. . Touitou, Y. (2012). Day and nighttime excretion of 6-sulphatoxymelatonin in adolescents and young adults with autistic disorder. Psychoneuroendocrinology, 37, 1990-1997. doi:10.1016/j.psyneuen.2012.04.013 
Vriend, J. L., Corkum, P. V., Moon, E. C., \& Smith, I. M. (2011). Behavioral interventions for sleep problems in children with autism spectrum disorders: Current findings and future directions. Journal of Pediatric Psychology,36, 1017-1029. doi:10.1093/jpepsy/jsr044

Weiskop, S., Matthews, J., \& Richdale, A. (2001). Treatment of sleep problems in a 5-year old boy with autism using behavioral principles. Autism, 5, 209-221. 


\section{Tables and Figures}

Table 1. A summary of participant characteristics at commencement of intervention

\begin{tabular}{|c|c|c|c|}
\hline Characteristics & Niko & Peter & Eric \\
\hline Age (Y-M) & $9-7$ & $14-6$ & $11-6$ \\
\hline Gender & Male & Male & Male \\
\hline Diagnosis & Asperger's syndrome & ASD & ASD \\
\hline VABS-II & & & \\
\hline Receptive and expressive & $2-5$ & $2-10$ & $5-6$ \\
\hline $\begin{array}{l}\text { language age equivalent } \\
(\mathrm{Y}-\mathrm{M})\end{array}$ & $4-11$ & $3-11$ & $12-3$ \\
\hline Educational environment & $\begin{array}{l}\text { Mainstream school } \\
\text { (teacher aide support) }\end{array}$ & Specialist school & Mainstream school \\
\hline GARS-3 & $\begin{array}{c}118 \\
\text { Very likely }\end{array}$ & $\begin{array}{c}108 \\
\text { Very likely }\end{array}$ & $\begin{array}{c}106 \\
\text { Very likely }\end{array}$ \\
\hline CBCL (6-18 years) & Clinical & Clinical & Clinical \\
\hline Medication & - & $\begin{array}{l}\text { Melatonin (3mg) } \\
\text { Risperidone }(0.25-0.5 \mathrm{mg}) \\
\text { Fluoxetine (20mg) }\end{array}$ & - \\
\hline
\end{tabular}


Table 2. Problem behavior, factors precipitating and/or maintaining behavior, hypothesized function, and parent and adolescent treatment components for all three participants

\begin{tabular}{|c|c|c|c|c|c|}
\hline & \multicolumn{2}{|c|}{ Niko } & \multicolumn{2}{|c|}{ Peter } & \multirow{2}{*}{$\frac{\text { Eric }}{\text { Delayed SOL }}$} \\
\hline & Frequent and prolonged NWs & Frequent EWs & Delayed SOL & Frequent EWs & \\
\hline $\begin{array}{l}\text { Factors thought to be } \\
\text { precipitating and/or } \\
\text { maintaining behavior }\end{array}$ & $\begin{array}{c}\text { Daytime sleeps; lack of } \\
\text { physiological sleep pressure; } \\
\text { electronic device use; } \\
\text { adolescent-reported discomfort } \\
\text { in bed; warm and comfortable } \\
\text { sleep-interfering environment }\end{array}$ & $\begin{array}{l}\text { Daytime sleeps; lack of } \\
\text { physiological sleep } \\
\text { pressure; electronic } \\
\text { device use; adolescent- } \\
\text { reported discomfort in } \\
\text { bed; warm and } \\
\text { comfortable sleep- } \\
\text { interfering environment }\end{array}$ & $\begin{array}{l}\text { Daytime sleeps; lack of } \\
\text { physiological sleep } \\
\text { pressure; inappropriate } \\
\text { sleep dependencies } \\
\text { (electronic device, bright } \\
\text { light); lack of } \\
\text { discriminative stimuli for } \\
\text { sleep; electronic device } \\
\text { use; exposure to bright } \\
\text { nightlight }\end{array}$ & $\begin{array}{l}\text { Daytime sleeps; lack of } \\
\text { physiological sleep } \\
\text { pressure; inappropriate } \\
\text { sleep dependencies } \\
\text { (electronic device, bright } \\
\text { light); lack of } \\
\text { discriminative stimuli for } \\
\text { sleep; electronic device } \\
\text { use; exposure to bright } \\
\text { nightlight }\end{array}$ & $\begin{array}{l}\text { Lack of physiological sleep } \\
\text { pressure; exposure to bright } \\
\text { light and stimulating content } \\
\text { on electronic devices; access } \\
\text { to food and drink; parent } \\
\text { responses to CC's; intrusive } \\
\text { internal stimuli; } \\
\text { hyperarousal }\end{array}$ \\
\hline Hypothesized function & $\begin{array}{l}\text { Tangible } \\
\text { Escape }\end{array}$ & $\begin{array}{l}\text { Tangible } \\
\text { Escape }\end{array}$ & Tangible & Tangible & $\begin{array}{c}\text { Tangible } \\
\text { Social attention } \\
\text { Escape (from intrusive } \\
\text { internal stimuli) }\end{array}$ \\
\hline $\begin{array}{l}\text { Parent treatment } \\
\text { components }\end{array}$ & $\begin{array}{l}\text { Sleep hygiene; bedtime fading } \\
\text { and sleep restriction } \\
\text { (elimination of naps); modified } \\
\text { extinction (removal of devices } \\
\text { at night and scheduled device } \\
\text { use); comfortable sleep setting; } \\
\text { positive reinforcement for } \\
\text { successive approximations } \\
\text { towards goal }\end{array}$ & $\begin{array}{l}\text { Sleep hygiene; bedtime } \\
\text { fading and sleep } \\
\text { restriction (elimination of } \\
\text { naps); modified extinction } \\
\text { (removal of devices at } \\
\text { night and scheduled } \\
\text { device use); comfortable } \\
\text { sleep setting; positive } \\
\text { reinforcement for } \\
\text { successive } \\
\text { approximations towards } \\
\text { goal; Gro-Clock }\end{array}$ & $\begin{array}{l}\text { Sleep hygiene; graduated } \\
\text { extinction (removal of } \\
\text { devices at night); bedtime } \\
\text { fading and sleep restriction } \\
\text { (elimination of naps); } \\
\text { Finished Box; sleep item; } \\
\text { replacement of nightlight; } \\
\text { consistent sleep cues; } \\
\text { positive reinforcement }\end{array}$ & $\begin{array}{l}\text { Sleep hygiene; graduated } \\
\text { extinction (removal of } \\
\text { devices at night); bedtime } \\
\text { fading and sleep } \\
\text { restriction (elimination of } \\
\text { naps); Finished Box; } \\
\text { sleep item; replacement of } \\
\text { nightlight; consistent } \\
\text { sleep cues; positive } \\
\text { reinforcement }\end{array}$ & $\begin{array}{l}\text { Restricted access to devices } \\
\text { after dinner; modified } \\
\text { extinction (minimal } \\
\text { engagement post-bedtime); } \\
\text { bedtime fading and sleep } \\
\text { restriction (set sleep and } \\
\text { wake times); positive } \\
\text { reinforcement }\end{array}$ \\
\hline $\begin{array}{l}\text { Adolescent treatment } \\
\text { components }\end{array}$ & $\begin{array}{l}\text { Psychoeducation; social story; } \\
\text { relaxation techniques }\end{array}$ & $\begin{array}{l}\text { Psychoeducation; social } \\
\text { story; relaxation } \\
\text { techniques }\end{array}$ & $\begin{array}{l}\text { Psychoeducation; social } \\
\text { story; relaxation } \\
\text { techniques }\end{array}$ & $\begin{array}{l}\text { Psychoeducation; social } \\
\text { story; relaxation } \\
\text { techniques }\end{array}$ & $\begin{array}{l}\text { Psychoeducation; relaxing } \\
\text { bedtime routine; sleep } \\
\text { checklist; relaxation } \\
\text { techniques; visualization }\end{array}$ \\
\hline
\end{tabular}


Table 3. Interobserver Agreement (IOA) between sleep diaries and videosomnography across target behaviors

\begin{tabular}{|c|c|c|c|c|c|c|}
\hline \multirow[b]{2}{*}{$\begin{array}{l}\text { Target } \\
\text { behavior }\end{array}$} & \multicolumn{2}{|c|}{ Niko } & \multicolumn{2}{|c|}{ Peter } & \multicolumn{2}{|c|}{ Eric } \\
\hline & Baseline & Intervention & Baseline & Intervention & Baseline & Intervention \\
\hline CCs & - & - & - & - & $91 \%$ & $100 \%$ \\
\hline SOL & - & - & $85 \%$ & $97 \%$ & $100 \%$ & $100 \%$ \\
\hline Duration NWs & - & $86 \%$ & $100 \%$ & $100 \%$ & - & - \\
\hline Duration EWs & - & $100 \%$ & $86 \%$ & $100 \%$ & - & - \\
\hline
\end{tabular}

Note. Lack of baseline sleep diary data for Niko inhibited calculation of IOA in this phase.

Table 4. Parent treatment fidelity

\begin{tabular}{llll}
\hline & Niko & Peter & Eric \\
\hline Treatment fidelity & $92 \%$ & $98 \%$ & $70 \%$ \\
\hline
\end{tabular}

Table 5. Pre- and post-treatment CSHQ scores

\begin{tabular}{lcc}
\hline & Niko & Eric \\
\hline Pre-treatment CSHQ & 52 & 58 \\
Post-treatment CSHQ & $44^{*}$ & $38^{* *}$ \\
\hline
\end{tabular}

Note. ${ }^{*}=$ Significant change. ${ }^{* *}=$ Clinical change.

Table 6. Post-treatment TARF-R scores

\begin{tabular}{lllllc}
\hline & Niko & & Peter & Eric & $\begin{array}{c}\text { Maximum } \\
\text { Score }\end{array}$ \\
\cline { 2 - 5 } Scale & Father & Mother & Father & Mother & \\
\hline Effectiveness & 21 & 21 & 21 & 21 & 21 \\
Reasonableness & 21 & 19 & 18 & 20 & 21 \\
Willingness & 21 & 18 & 16 & 17 & 21 \\
Cost & 14 & 10 & 14 & 14 & 14 \\
Negative side-effects & 21 & 15 & 15 & 15 & 21 \\
Disruption/time & 15 & 18 & 16 & 18 & 21 \\
Problem severity* & 10 & 11 & 11 & 2 & 14 \\
Understanding of treatment* & 7 & 7 & 7 & 7 & 7 \\
Total acceptability & 113 & 98 & 94 & 105 & 119 \\
\hline
\end{tabular}

Note. * Not included in total acceptability score 


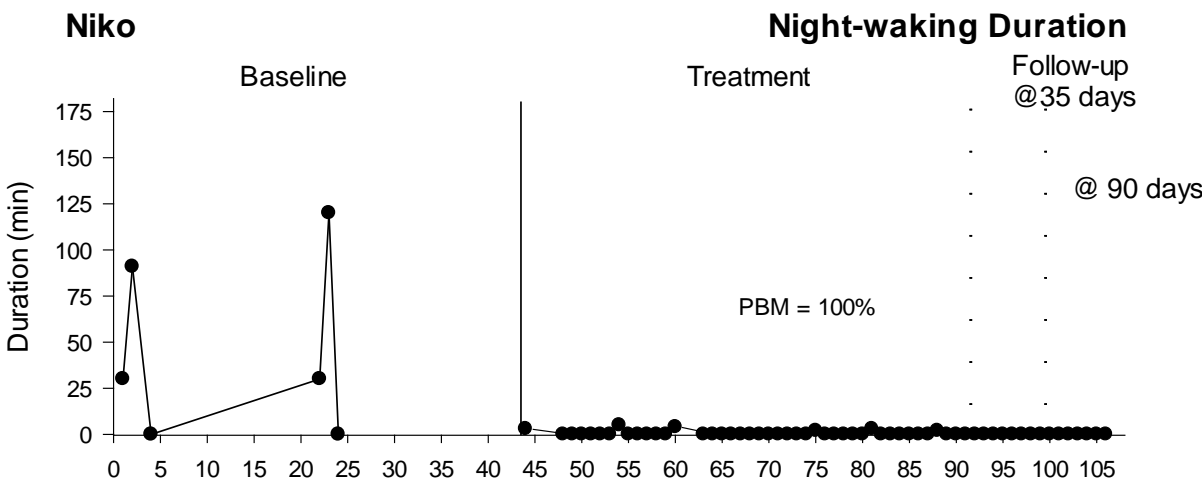

\section{Early Waking Duration}

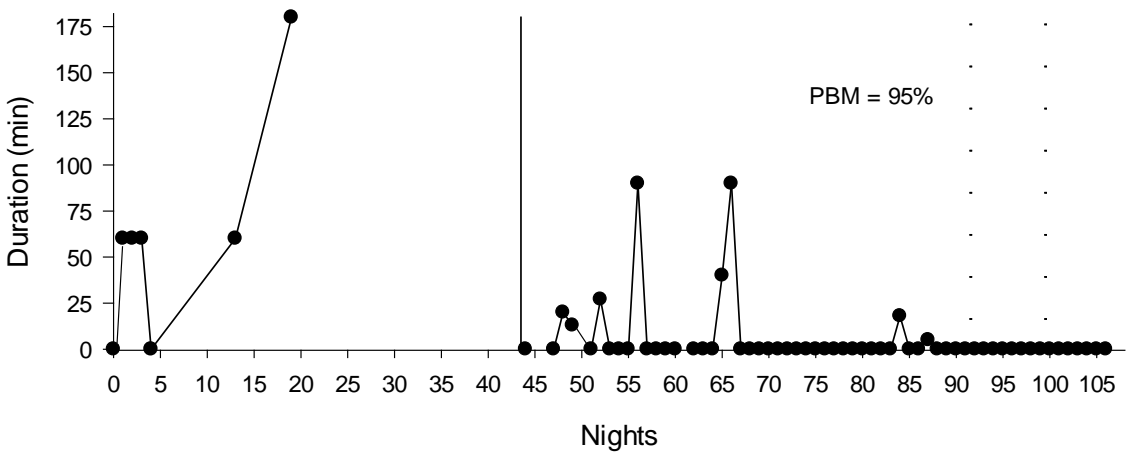

Fig. 1 Sleep outcomes for Niko: Duration of NWs and EWs across baseline, intervention, and follow-up phases 

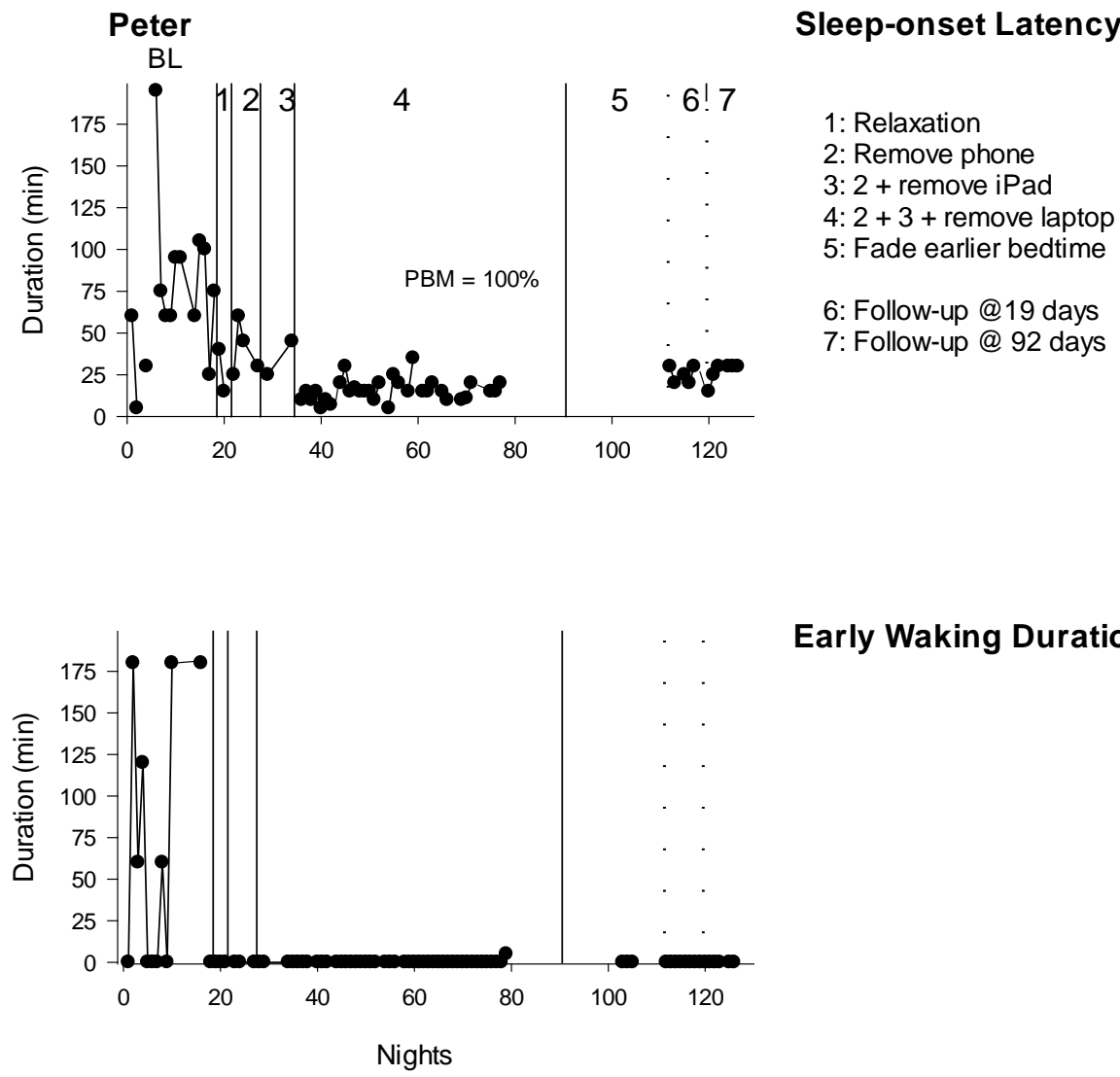

\section{Early Waking Duration}

Fig. 2 Sleep outcomes for Peter: SOL and duration of EWs across baseline, intervention, and follow-up phases 

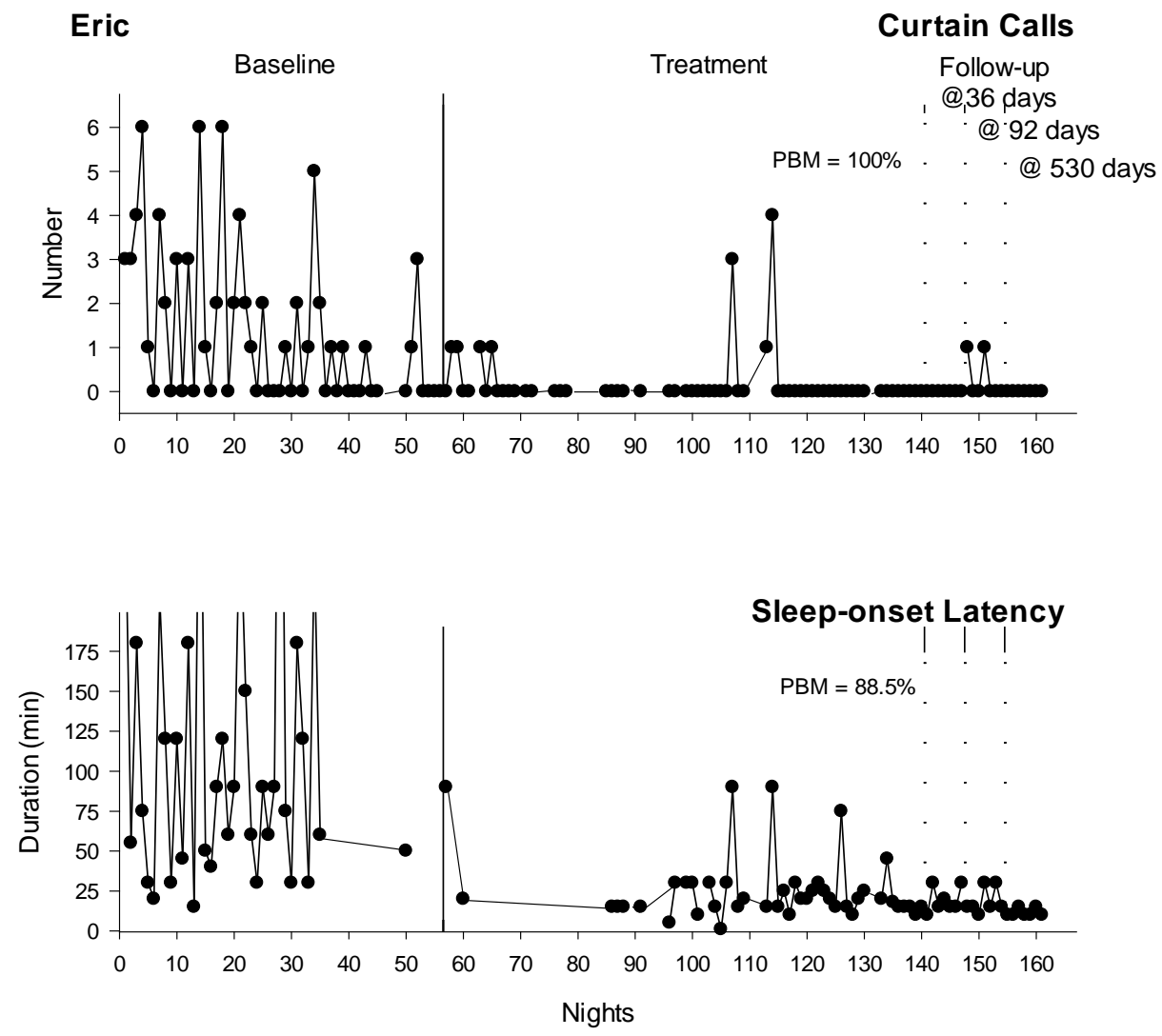

Fig. 3 Sleep outcomes for Eric: CCs and SOL across baseline, intervention, and follow-up phases 